\title{
Optimal compression for mixed signal states
}

\author{
Michał Horodeckit \\ Institute of Theoretical Physics and Astrophysics \\ University of Gdańsk, 80-952 Gdańsk, Poland
}

We consider the problem of the optimal compression rate in the case of the source producing mixed signal states within the visible scheme (where Alice, who is to compress the signal, can know the identities of the produced states). We show that a simple strategy based on replacing the signal states with their extensions gives optimal compression. As a result we obtain a considerable simplification of the formula for optimal compression rate within visible scheme.

Pacs Numbers: 03.67.-a

Given a quantum source, what is the minimal number of quantum resources needed for faithful transmission of the states produced by the source? This quantum analogue of the problem of data compression [1] was stated for the first time by Schumacher [2]. It has been solved for stationary memoryless sources generating pure states 24. In general, the stationary memoryless sources are described by ensemble $\left\{p_{i}, \varrho_{i}\right\}$. This means that the source emits system in state $\varrho_{i}$ with probability $p_{i}$ (one can of course generalize it by considering probability measure on the set of states). The lack of memory implies that the state of the sequence of systems emitted by the source is in product state $\varrho_{i_{1}} \otimes \ldots \otimes \varrho_{i_{N}}$ and probability of emission of such string is product of suitable probabilities $p_{i_{1}} \ldots p_{i_{N}}$. Henceforth we will deal with such kind of sources.

It appears, that for pure signal states $\varrho_{i}$, the minimal number of qubits allowing faithful recovery of the input states is equal to the von Neumann entropy of the density matrix of ensemble $\varrho=\sum_{i} p_{i} \varrho_{i}$ [5]. Then the von Neumann entropy has clear interpretation within purely quantum communication theory.

In the case of $\varrho_{i}$ being impure the problem is unsolved. Apart from some particular cases we do not know much about optimal compression of ensembles of mixed states. The scheme that succeeded in the pure states case can be also applied in the present case, compressing the signal down to the von Neumann entropy. However, one knows that in many cases it is not optimal compression [6]. A good candidate for the minimal number of qubits in this general case could be the Levitin-Holevo function [7] of ensemble $I_{L H}=S\left(\sum_{i} p_{i} \varrho_{i}\right)-\sum_{i} p_{i} S\left(\varrho_{i}\right)$ where $S$ is von Neumann entropy. Indeed, in this quantity the lost of information caused by impurity of signal states is taken into account by subtracting their mean entropy. As a matter of fact, it has been proven [8,9] that $I_{L H}$ is lower bound for the needed number of qubits. However a very difficult problem, whether this rate of compression can be reached remains still unsolved. Additional motivation to consider this problem is that one would like to know whether $I_{L H}$ has interpretation in terms of qubits (so far it has the interpretation in terms of capability of sending classical bits via quantum states 10 12]).

In general, the scheme of compression is as follows. Alice (who is to compress the signal) waits for a long sequence of the systems generated by the source. Then she performs some operation on the sequence. Her aim is to decrease the support of the total density matrix of the ensemble of sequences (the least Hilbert space the matrix lives on), as the number of needed qubits is equal to the logarithm of the dimension of the support. However, she must do it in a clever way, in order not to disturb the signal too much, so that Bob would be able to recover it with high fidelity.

There are two basic schemes of compression. In the first one, called blind, Alice does not know the identities of the produces states. Then all she can do is to apply some quantum operation, independent of the input states. If, instead, she knows the identities of the states (visible scheme) her operation can be state-dependent, so that she has more possibilities. Of course, Bob does not know the identities of signal states in either case, so that his operation is always state-independent. Thus, in general, the compression could be better within the visible scheme. The optimal compression rate for pure signal states appears to be independent of the kind of applied scheme [4]. For mixed state case, the answer is not known. In both schemes to obtain the optimal compression rate, one must perform optimization over Alice and Bob actions satisfying the condition of high fidelity of transmission. All that must be performed in the limit of long sequences. Thus the task is exceedingly difficult.

In one of the attempts to solve the problem, a simple visible protocol of compression was proposed [13,8]. Namely, Alice can replace the signal states [15] with their purifications [14], applying then Schumacher (or JozsaSchumacher [3]) compression protocol to the resulting ensemble of pure states. More generally 80 Alice can replace signal states with their extensions (by extension of a state $\sigma$ we mean another state that, partially traced, reproduces $\sigma$ ) that are not necessarily pure. This replacement aims at decreasing the von Neumann entropy $S$ of the density matrix of the initial ensemble to some lower value $S^{\prime}$. If it is possible, then the subsequent JozsaSchumacher (JS) protocol will compress the signal at rate $S^{\prime}$ qubits/message, hence with better performance than in the case of direct application of the protocol, resulting in $S$ qubits/message. 
In this paper we consider visible scheme. We prove that the above very simple strategy provides optimal compression rate. More precisely, to compress the signal optimally, Alice should replace the sequences of signal states with their extensions chosen in such a way that the von Neumann entropy of the resulting ensemble is minimal. As a result, we obtain a considerable simplification of the formula for optimal compression rate. The very tedious task of optimization is now reduced to minimization of the von Neumann entropy of the ensemble of extensions.

Let us now introduce some notation (the same as in Ref. [8]). Suppose that the source generates a system in state $\varrho_{i}^{0}$ acting on a Hilbert space $\mathcal{H}_{\mathcal{Q}}$ with probability $p_{i}^{0}$. The produced ensemble $\mathcal{E}_{0}=\left\{p_{i}^{0}, \varrho_{i}^{0}\right\}$ has the density matrix $\varrho^{0}=\sum_{i} p_{i}^{0} \varrho_{i}^{0}$. Denote the product $\varrho_{i_{1}}^{0} \otimes \ldots \otimes \varrho_{i_{N}}^{0}$ by $\varrho_{i}$, where $i$ stands now for multi-index (to avoid complicated notation we do not write the index $N$ explicitly unless necessary). The corresponding ensemble and state are denoted by $\mathcal{E}$ and $\varrho$ respectively. Now Alice performs a coding operation over the initial ensemble $\mathcal{E}$ ascribing to any input state $\varrho_{i}$ a new state $\tilde{\varrho}_{i}$. The map $\varrho_{i} \rightarrow \varrho_{i}=\Lambda_{A}\left(\varrho_{i}\right)$ is supposed to be a quantum operation i.e. linear completely positive trace-preserving map for blind scheme or an arbitrary map - for visible one. In the latter case we allow Alice to know which states are generated by the source, so that she can prepare separately each of the states $\tilde{\varrho}_{i}$ for each $i$.

The new states $\tilde{\varrho}_{i}$ represent the compressed signal that is then flipped onto the suitable number of qubits determined by the dimension of subspace occupied by the total state $\tilde{\varrho}$ of the ensemble and sent through the noiseless channel to Bob. Now the states $\tilde{\varrho}_{i}$ are to be decompressed to become close to the initial states $\varrho_{i}$. For this purpose Bob performs some established quantum operation $\Lambda$ which of course does not depend on $i$. Then the resulting states are $\varrho_{i}^{\prime}=\Lambda_{B}\left(\tilde{\varrho}_{i}\right)$ and the total scheme is the following

$$
\begin{array}{ll}
\varrho_{i} \underset{\Lambda_{A}}{\stackrel{\text { compression }}{\longrightarrow}} \tilde{\varrho}_{i} \underset{I}{\stackrel{\text { noiseless channel }}{\longrightarrow}} \tilde{\varrho}_{i} \\
\underset{\Lambda_{B}}{\operatorname{decompression}}{ }^{\longrightarrow} & \varrho_{i}^{\prime},
\end{array}
$$

where $\varrho_{i}$ and $\varrho_{i}^{\prime}$ act on the Hilbert space $\mathcal{H}_{Q}^{\otimes N}$ while $\tilde{\varrho}_{i}$ on the channel Hilbert space $H_{\mathcal{C}}$. Now we should determine the measure of quality of transmission $\varrho_{i} \rightarrow \varrho_{i}^{\prime}$. As one knows, there exist many different metrics on the set of mixed states. The most common ones are HilbertSchmidt distance $D_{H-S}^{2}(\varrho, \sigma)=\operatorname{Tr}(\varrho-\sigma)^{2}$, the one induced by trace norm $\|\varrho-\sigma\|=\operatorname{Tr}|\varrho-\sigma|$ and the Bures metric $D_{B}=2-\sqrt{F(\varrho, \sigma)}$, where the fidelity $F$ [16, 17 is given by

$$
F(\varrho, \sigma)=[\operatorname{Tr}(\sqrt{\sqrt{\varrho} \sigma \sqrt{\varrho}})]^{2} .
$$

Instead of Bures metric, one usually uses directly the fidelity. The latter has an appealing property: if one of the states (say $\varrho$ ) is pure then it is of the form

$$
F(\sigma,|\psi\rangle\langle\psi|)=\langle\psi|\sigma| \psi\rangle
$$

In this case the fidelity has a clear interpretation as probability that the state $\sigma$ passes the test of being $\psi$. The fidelity was used in the problem of compression of quantum information [2 2 , and it is now an important tool in quantum information theory. However some results were obtained by use of other measures of quality of transmission. To the author knowledge, there is no special discrepancy among the results obtained via different metrics. In fact, it is yet not clear whether and to what extent different metrics could lead to non-equivalent conclusions. In this paper we will use fidelity, partially due to one of its properties being especially useful in the context of the problem of extensions we are dealing with. Namely [16,17 the fidelity can be expressed in the following way

$$
F(\sigma, \varrho)=\max _{\psi}|\langle\psi \mid \phi\rangle|^{2},
$$

where $\phi$ is arbitrary purification of $\sigma$ and the maximum runs over all possible purifications of $\varrho$. As we will see further, this property allows to prove an important lemma.

Consequently, the average fidelity $\bar{F}\left(\mathcal{E}, \mathcal{E}^{\prime}\right) \equiv$ $\sum_{i} p_{i} F\left(\varrho_{i}, \varrho_{i}^{\prime}\right)$ will indicate us the quality of the process of recovery of quantum information by Bob after compression by Alice. Now, for a fixed source determined by the ensemble $\mathcal{E}_{0}$, one considers the sequence of compression-decompression pairs $\left(\Lambda_{A}, \Lambda_{B}\right)$ with the property that

$$
\lim _{N \rightarrow \infty} \bar{F}\left(\mathcal{E}, \mathcal{E}^{\prime}\right)=1
$$

(recall that the pair is implicitly indexed by N). Such sequences will be called protocols.

Define now the quantity $R_{P}$ characterizing the asymptotic degree of compression of the initial quantum data at a given protocol $P$ by

$$
R_{P}=\lim _{N \rightarrow \infty} \frac{1}{N} \log \operatorname{dim} \tilde{\varrho}
$$

Here $\operatorname{dim} \tilde{\varrho}$ denotes the dimension of the support of the state $\tilde{\varrho}$ given by the number of its nonzero eigenvalues. The quantity $\log \operatorname{dim} \tilde{\varrho}$ has the interpretation of the num-

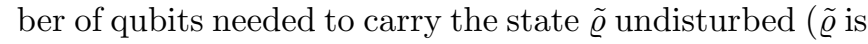
to be transferred through a noiseless channel). Actually, only one of the signal sequences $\tilde{\varrho}_{i}$ is being transmitted at a time. However, it is easy to see, that $\operatorname{dim} \tilde{\varrho}$ is the minimal dimension that guarantees transmission of any of the states $\tilde{\varrho}_{i}$ without disturbance.

Now, given a class $\mathcal{P}$ of protocols, we define the quantity

$$
I_{\mathcal{P}}=\inf _{P \in \mathcal{P}} R_{P}
$$


which is equal to the least number of qubits per system needed for asymptotically faithful transmission of the initial signal states from Alice to Bob within the considered class of protocols (to be strict one needs $I_{\mathcal{P}}+\delta$ qubits per message, where $\delta$ can be chosen arbitrarily small). Now, if $\mathcal{P}$ is the set of visible protocols, the $I_{\mathcal{P}}$ is called effective information carried by the ensemble [8] and is denoted by $I_{\text {eff }}$ (optimal rate within the class of blind protocols is called passive information). As one can see, the definition of the effective information, even though physically natural, is very complicated from mathematical point of view. One must optimize the limit (6) over the Alice and Bob actions keeping satisfied the condition (5) at the same time. Moreover, the definition does not give any intuition on how the structure of ensemble could be related to its effective information content.

Let us now try to reduce the problem to obtain more transparent form of the effective information. Consider the most general compression-decompression protocol. Any Bob's operation, as a completely positive tracepreserving map, amounts to (i) adding ancilla in some pure state, (ii) performing unitary transformation over the total system and (iii) performing partial trace [18]. Now, the two first stages can be incorporated into Alice action. Then decompression will amount only to performing partial trace. Of course, the new protocol will give the same rate of compression as the previous one, because both stages do not change the dimension of the support of a state. Thus we can consider optimal protocol in the following form

$$
\varrho_{i} \underset{\Lambda_{A}}{\stackrel{\text { compression }}{\longrightarrow}} \varrho_{i}^{\prime e x t} \stackrel{\text { decompression }}{\underset{\Lambda_{B}=\operatorname{Tr}_{a n c}}{\longrightarrow}} \varrho_{i}^{\prime}
$$

where $\varrho_{i}^{\prime e x t}$ are some extensions of the state $\varrho_{i}^{\prime}$ and they act on the Hilbert space $\mathcal{H}_{Q}^{\otimes N} \otimes \mathcal{H}_{\text {anc }}$ (in the following, the extensions of a state $\sigma$ will be denoted by $\left.\sigma^{\text {ext }}\right)$. Then the optimal compression rate is given by 19

$$
I_{e f f}=\lim _{n} \frac{1}{N} \log \operatorname{dim} \varrho^{\prime e x t} .
$$

We will need the following lemma.

Lemma. Let $\varrho, \varrho^{\prime}$ act on space $\mathcal{H}_{Q}^{\otimes N}$ and let $\varrho^{\prime e x t}$, acting on $\mathcal{H}_{Q}^{\otimes N} \otimes \mathcal{H}_{\text {anc }}$ be extension of $\varrho^{\prime}$. Then there exists a state $\varrho^{\text {ext }}$ acting on $\mathcal{H}_{Q}^{\otimes n} \otimes \mathcal{H}_{\text {anc }}$ such that (a) $\varrho^{e x t}$ is an extension of $\varrho$ (b) $F\left(\varrho^{e x t}, \varrho^{\prime e x t}\right)=F\left(\varrho, \varrho^{\prime}\right)$.

Proof. Let $\mathcal{H}_{\text {ext }}=\mathcal{H}_{Q}^{\otimes N} \otimes \mathcal{H}_{a n c}$ and let $\phi^{\prime} \in$ $\mathcal{H}_{\text {ext }} \otimes \mathcal{H}_{\text {pur }}$ be purification of $\varrho^{\prime \text { ext }}$. Then it is also purification of the state $\varrho^{\prime}$. From the formula (里) we obtain that there exists some purification $\phi$ of $\varrho$ such that $F\left(\varrho, \varrho^{\prime}\right)=\left|\left\langle\phi^{\prime} \mid \phi\right\rangle\right|^{2}$. Now we can take $\varrho^{\text {ext }}=$ $\operatorname{Tr}_{\mathcal{H}_{\text {pur }}}|\phi\rangle\langle\phi|$. Using the formula (1) once more, we get $F\left(\varrho^{e x t}, \varrho^{\prime e x t}\right) \geq\left|\left\langle\phi^{\prime} \mid \phi\right\rangle\right|^{2}=F\left(\varrho, \varrho^{\prime}\right)$. Since the fidelity does not decrease under partial trace 20] (this can be easily seen from (4) $)$, we obtain $F\left(\varrho^{e x t}, \varrho^{\text {ext }}\right)=F\left(\varrho, \varrho^{\prime}\right)$.

Let us now formulate the main result of the paper.
Theorem. Let $\mathcal{E}^{e x t}=\left\{p_{i}, \varrho_{i}^{\text {ext }}\right\}$ with $\varrho_{i}^{\text {ext }}$ being extensions of the signal states $\varrho_{i}$; let $\varrho^{\text {ext }}$ be the total density matrix of ensemble $\mathcal{E}^{e x t}$. Then the optimal compression rate within the visible scheme is given by

$$
I_{e f f}=\lim _{N \rightarrow \infty} \frac{1}{N} \inf S\left(\varrho^{e x t}\right)
$$

where the infimum runs over the set of ensembles $\mathcal{E}^{\text {ext }}$.

Remarks. (1) Since one can choose trivial $\mathcal{H}_{\text {anc }}$ $\left(\mathcal{H}_{\text {anc }}=\mathcal{C}\right), \varrho_{i}$ itself is the extension of $\varrho_{i}$, too. (2) One can show that the limit on the right hand side of the equality (10) exists. Indeed, it follows from the fact that if a sequence $\left\{a_{n}\right\}$ satisfies $a_{n} \leq k n$ for some $k$ and $a_{n}+a_{m} \geq a_{n+m}$ for any $m, n$, then $a_{n} / n$ is convergent 21 .

Proof. To prove that the formula (10) is valid, we must first provide the protocol that achieves such rate, and then show that the latter is equal to the optimal rate given by (9).

To this end, consider the following concatenated protocol (cf. Ref. \&) (call it extension protocol and denote by $E P)$. Alice replaces the signal state with the state $\varrho_{i}^{e x t}$, and then applies the JS protocol [3]. The number of needed qubits per system is now equal to the entropy of the density matrix of the new ensemble. As JS protocol needs no decompression [3], Bob has only to perform partial trace to come back to the original space $\mathcal{H}_{Q}^{\otimes N}$. Then, the overall scheme is the following

$$
\begin{aligned}
& \varrho_{i_{1}} \otimes \ldots \otimes \varrho_{i_{k}} \stackrel{\substack{\text { Alice's } \\
\text { action }}}{\longrightarrow} \varrho_{i_{1}}^{e x t} \otimes \ldots \otimes \varrho_{i_{k}}^{e x t} \longrightarrow
\end{aligned}
$$

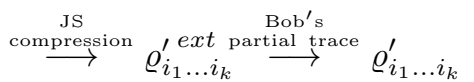

Here $i_{j}$ 's are multi-indices of length $N ; \varrho_{i_{1}} \otimes \ldots \otimes \varrho_{i_{k}}$ and $\varrho_{i_{1} \ldots i_{k}}^{\prime}$ act on the Hilbert space $\left(\mathcal{H}_{Q}^{\otimes N}\right)^{\otimes k}$ while $\tilde{\varrho}_{i_{1}}^{\text {ext }} \otimes \ldots \otimes \tilde{\varrho}_{i_{k}}^{\text {ext }}$ and $\tilde{\varrho}_{i_{1} \ldots i_{k}}^{\text {ext }}$ act on $\left(\mathcal{H}_{Q}^{\otimes N} \otimes \mathcal{H}_{a n c}\right)^{\otimes k}$. The former two states can be obtained from the latter ones by tracing over the space $\mathcal{H}_{a n c}^{\otimes k}$. Now, as it was mentioned, the fidelity does not decrease under partial trace. Then (as in Ref. [8] for different transmission quality measure) we obtain that average fidelity produced by the composed protocol is greater than or equal to the one within the "intermediate" JS compression protocol. The latter fidelity tends to one if $N$ is kept fixed and $k$ tends to infinity (of course, $N$, although fixed, can be chosen arbitrarily large). Thus the total protocol satisfies asymptotic fidelity condition (5).

Since the JS protocol compresses the signal down to the von Neumann entropy of the ensemble, the extension protocol has compression rate equal to

$$
R_{E P}=\lim _{N \rightarrow \infty} \frac{1}{N} S\left(\varrho^{e x t}\right)
$$

Minimizing this expression over all possible extensions of signal states we obtain the rate of optimal extension protocol (OEP) 


$$
R_{O E P} \equiv \inf _{E P} R_{E P}=\lim _{N \rightarrow \infty} \frac{1}{N} \inf S\left(\varrho^{e x t}\right) .
$$

Now we must show that $R_{O E P} \leq I_{\text {eff }}$. To this end consider the optimal protocol of the form (8) so that $I_{\text {eff }}$ is given by equation (9). Then it suffices to find such extensions $\varrho_{i}^{\text {ext }}$ that

$$
\lim _{N \rightarrow \infty} \frac{1}{N} S\left(\varrho^{e x t}\right) \leq \lim _{N \rightarrow \infty} \frac{1}{N} \log \operatorname{dim} \varrho^{\prime e x t} .
$$

The suitable extensions are suggested by the lemma. Namely, suppose that $N$ is large, so that $F\left(\mathcal{E}, \mathcal{E}^{\prime}\right)>1-\epsilon$ (within the considered optimal protocol). Then, by the lemma there exist extensions $\varrho_{i}^{\text {ext }}$ of $\varrho_{i}$ such that $\bar{F}\left(\mathcal{E}^{e x t}, \mathcal{E}^{\prime e x t}\right)>1-\epsilon$. Now we can use the inequality proved in 22] (which is similar to the Fannes inequality [23) saying that for states acting on Hilbert space $\mathcal{H}$ we have

$$
\left|S(\varrho)-S\left(\varrho^{\prime}\right)\right| \leq 2 \log \operatorname{dim} \mathcal{H} \sqrt{1-F\left(\varrho, \varrho^{\prime}\right)}+1
$$

if only $F\left(\varrho, \varrho^{\prime}\right)>1-\frac{1}{36}$. By double concavity of square of $F$ [9] we obtain in our case that

$$
\begin{aligned}
& \frac{1}{N}\left|S\left(\varrho^{e x t}\right)-S\left(\varrho^{\prime e x t}\right)\right| \leq \\
& 4\left(\log \operatorname{dim} \mathcal{H}_{\mathcal{Q}}+\frac{1}{N} \log \operatorname{dim} \mathcal{H}_{\text {anc }}\right) \sqrt{\epsilon}+\frac{1}{N}
\end{aligned}
$$

One can show 24] that it suffices to consider $\mathcal{H}_{\text {anc }}$ satisfying $\log \operatorname{dim} \mathcal{H}_{a n c} \leq 2 N \log \operatorname{dim} \mathcal{H}_{Q}$. Thus the entropy (per system) of the state $\varrho^{e x t}$ is asymptotically equal to the one of $\varrho^{\prime e x t}$. Now, since $S\left(\varrho^{\prime e x t}\right) \leq \log \operatorname{dim} \varrho^{\prime e x t}$ we obtain the inequality (14).

To summarize, we obtained much simpler formula for optimal compression rate in visible coding scheme. So far the task was to minimize the support of the states under Alice and Bob operations constrained by the asymptotic high fidelity condition. The latter is very difficult to deal with in the case of mixed states. The present expression does not involve such constraint, nor it involves Alice and Bob actions. Now one needs minimize entropy (which is more feasible than dealing with dimension of the support) varying over extensions of the ensemble. Thus the constraints are now much more convenient. An interesting question arises: Can the optimal compression be achieved by means of pure extensions (purifications)? A closely related question is: Given an ensemble, do there exist such purifications, that the entropy of the purification ensemble is not greater than the entropy of the initial one? If it is not the case in general, can it be asymptotically true for typical sequences of states? Finally, one could ask, whether the limit in the formula (10) is really needed. It might be the case that the minimal entropy could be attained by means of extensions of single signals $\varrho_{i}^{0}$. However, almost everywhere in quantum information theory the collective operations are much more powerful than the ones performed on separate systems. Then it is likely, that collective extensions of long signal sequences are necessary to obtain optimal compression.

We believe that the presented result will stimulate to answer these questions, to find whether the LevitinHolevo function has physical sense in terms of quantum bits, and eventually, to resolve the highly nontrivial problem of compression of quantum information carried by ensembles of mixed states.

\section{ACKNOWLEDGMENTS}

The author is grateful to Ryszard Horodecki for stimulating discussion and helpful comments. He also would like to thank Chris Fuchs for helpful discussion. The work is supported by Polish Committee for Scientific Research, Contract No. 2P03B 14317.

* $\quad$ E-mail address: michalh@iftia.univ.gda.pl

[1] E. Shannon, Bell Syst. Tech. J. 27, 379 (1948).

[2] B. Schumacher, Phys. Rev. A 51, 2738 (1995).

[3] R. Jozsa and B. Schumacher, J. Mod. Opt. 41, 2343 (1994).

[4] H. Barnum, C. A. Fuchs, R. Jozsa and B. Schumacher, Phys. Rev. A 54, 4707 (1996).

[5] This result was generalized to cover the case of unknown parameters of the source, see M. Horodecki, R. Horodecki and P. Horodecki, Acta Phys. Slovaca 48, 133 (1998); R. Jozsa, M. Horodecki, P. Horodecki and R. Horodecki, Phys. Rev. Lett. 81, 1714 (1998). See also C. Krattenthaler and P. Slater quant-ph/9612043; M. Nielsen PhD thesis, University of New Mexico (1998).

[6] Hoi-Kwong Lo, Opt. Commun. 119, 552 (1995); R. Jozsa, unpublished.

[7] A. S. Holevo, Probl. Peredachi Inform. 8, 63 (1973).

[8] M. Horodecki, Phys. Rev. A 57, 3364 (1998).

[9] H. Barnum, C. Caves, C. A. Fuchs, R. Jozsa and B. Schumacher (unpublished); C. A. Fuchs and J. van de Graaf, IEEE Trans. IT 45, 1216 (1999).

[10] P. Hauslanden, R. Jozsa, B. Schumacher, M. Westmoreland and W. K. Wooters, Phys. Rev. A 54, 1869 (1996).

[11] A. S. Holevo, IEEE Trans. IT 44, 269 (1998); also available as e-print quant-ph/9708046;

[12] B. Schumacher and M. Westmoreland Phys. Rev. A 56, 131 (1997).

[13] H. Barnum, C. Caves, C. Fuchs, R. Jozsa and B. Schumacher (unpublished).

[14] Recall that the purification of a state $\varrho$ acting on $\mathcal{H}$ is a pure state $P$ acting on $\mathcal{H} \otimes \mathcal{H}^{\prime}$ such that $\varrho$ is its partial trace over the space $\mathcal{H}^{\prime}$.

[15] By signal state we mean the state of sequence of the systems emitted by the source.

[16] R. Jozsa, J. Mod. Opt. 41, 2315 (1994).

[17] A. Uhlmann, Rep. Math. Phys. 9, 273 (1976). 
[18] K. Kraus, States, Effects and Operations: Fundamental Notions of Quantum Theory, Wiley, New York, 1991.

[19] The presented reasoning is also true within the blind scheme. Then the formula (9) holds for passive information, too (although the involved extensions may be different).

[20] In fact, one has $F\left(\sigma, \sigma^{\prime}\right) \leq F\left(\Lambda(\sigma), \Lambda\left(\sigma^{\prime}\right)\right)$ for any tracepreserving completely positive map $\Lambda$ (see H. Barnum, C. M. Caves, C. A. Fuchs, R. Jozsa and B. Schumacher Phys. Rev. Lett. 76, 2818 (1996)).

[21] See e.g. H. Barnum, M. Nielsen and B. Schumacher Phys. Rev. A 57, 4153 (1998).

[22] H. Barnum, J. Smolin, and B. Terhal Phys. Rev. A 58, 3496 (1998).

[23] M. Fannes, Commun. Math. Phys. 31, 291 (1973); see also M. Ohya and D. Petz, Quantum Entropy and Its Use, p. 22, Springer-Verlag 1993.

[24] M. Horodecki, in preparation. 